\title{
Heartbeat: Inflammatory biomarkers in atrial fibrillation
}

\section{Catherine M Otto}

The association of systemic inflammation with atrial fibrillation and a prothrombotic state has received increasing attention recently, but whether this degree of inflammation provides additive value for prediction of clinical outcomes in adults with atrial fibrillation has not been established. In this issue of Heart, Hijazi and colleagues (see page 508) measured inflammatory biomarkers in over 14 thousand patients with atrial fibrillation in the Apixaban for Reduction In Stroke and Other Thromboembolic Events in Atrial Fibrillation (ARISTOTLE) trial. Cox regression was used to assess the relationship between outcomes at a mean follow-up of 1.9 years and baseline quartile groups of interleukin 6 (IL-6) and C reactive protein (CRP) levels. In these patients with atrial fibrillation who were anti-coagulated with apixaban or warfarin, IL-6 and CRP levels were not associated with an increased risk of stroke or systemic embolism and did not provide additive value to currently utilized risk scores. In contrast, both inflammatory biomarkers were significantly associated with an increased risk for all-cause mortality - the hazard ratio (HR) for IL-6 was 1.93 (1.57 to 2.37) and for CRP was 1.49 (1.24 to 1.79 ) when comparing the highest and lowest quartiles (figure 1), even after adjustment for clinical factors and other cardiovascular biomarkers.

The association of atrial fibrillation and systemic inflammation has clinical implications beyond predicting risk of adverse outcomes. If the risk of systemic embolization is simply related to the mechanical dysfunction of the left atrium, with atrial thrombus due to stasis of blood flow, then mechanical interventions such as occluding the left atrial appendage or electrical interventions to restore normal atrial contraction should effectively prevent systemic embolic events. Conversely, if the risk of stroke is related to systemic inflammation, endothelial dysfunction and a pro-thrombotic state, then mechanical or electrical interventions may not be enough to prevent adverse events. Based on the data presented in this study, Okaumura concludes in an accompanying

Correspondence to Professor Catherine M Otto, Division of Cardiology, University of Washington, Seattle, WA 98195, USA; cmotto@u.washington.edu

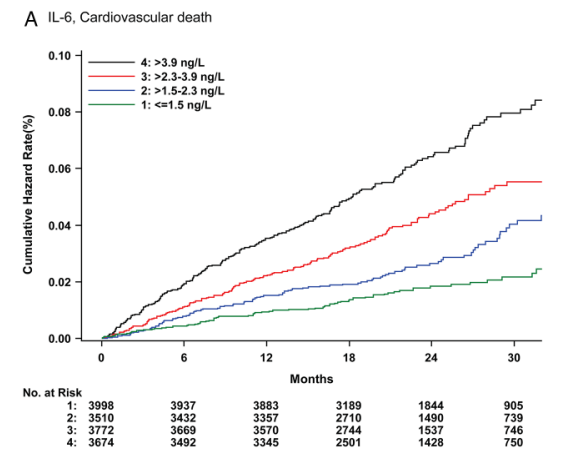

B CRP, Cardiovascular death

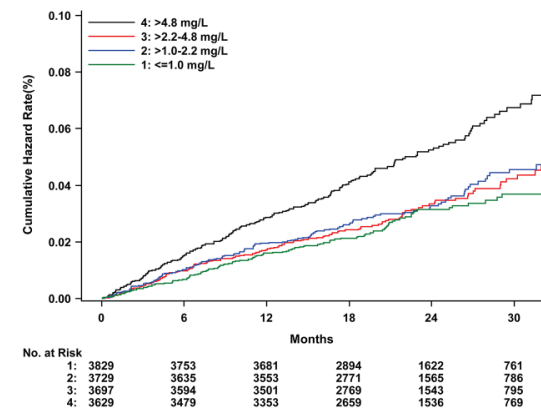

Figure 1 Cumulative hazard rates for cardiovascular mortality according to quartile groups at baseline of IL- $6(A)$ and $C$ reactive protein (CRP) (B).

editorial (see page 487): "in the setting of $\mathrm{AF}$, these cardiac biomarkers may in part reflect the atrial dysfunction resulting from fibrosis and wall stretch, possibly leading to the risk of thromboembolism. These observations suggest that, with hypercoagulability controlled by anticoagulants, left atrial blood stasis ('flow abnormalities') due to cardiac (probably atrial) dysfunction was the main contributor to the prothrombotic state in these patients; the role of inflammation may be small. In other words, the inflammatory status in AF may simply be the consequence of cardiac conditions summarised by the CHA2DS2-VASc score or, though infrequently, of underlying malignancies or COPD".

Accurate knowledge of normal cardiovascular changes during pregnancy is essential for management of pregnant women with heart disease, yet our current understanding is based on very small datasets, with less than 20 to up to 100 subjects in any one publication. In an effort to better define the normal time course and magnitude of the increase in cardiac output with pregnancy, Meah and colleagues (see page 518) performed a series of meta-analyses using 39 published studies resulting in pooled sample sizes of 259 748 for each hemodynamic parameter. Cardiac output was measured by echocardiography in most of these studies, with a minority using impedance cardiography
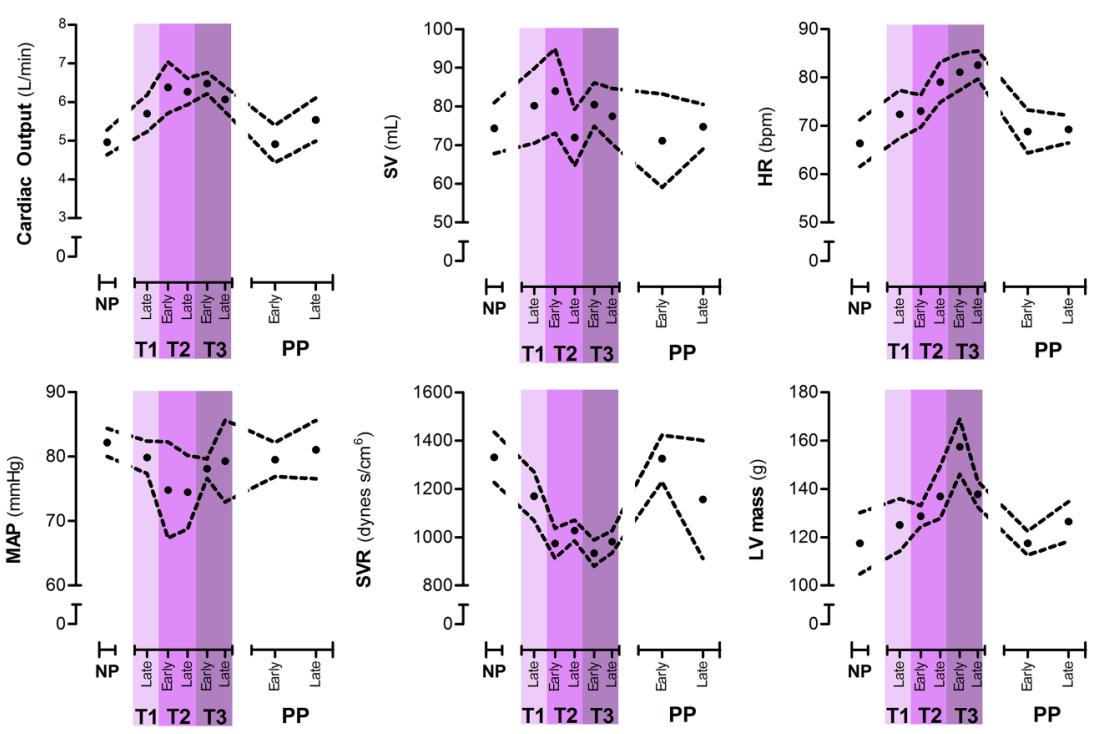

Figure 2 Compiled weighted mean and 95\% Cls derived from meta-analyses for cardiac output, heart rate (HR), stroke volume (SV), mean arterial pressure (MAP), systemic vascular resistance (SVR) and left ventricular (LV) mass at each gestational age. Coloured bars represent the first, second and third trimester of gestation. NP, non-pregnant; T1, trimester one; T2, trimester two; $\mathrm{T} 3$, trimester three; PP, postpartum. 


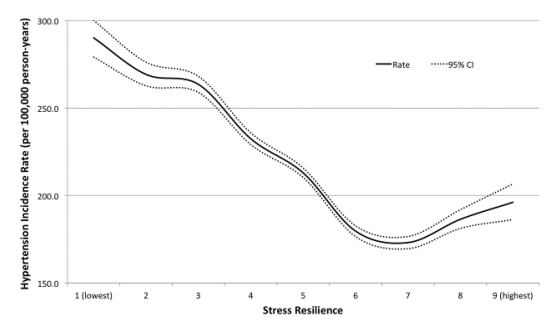

Figure 3 Hypertension incidence rates in adult men (median attained age 47 years, maximum 62 ) by stress resilience at age 18 years.

or inert gas re-breathing methods. This combined data indicates that cardiac output rises in a non-linear fashion in the first and second trimesters, with a peak $31 \%$ over non-pregnant values early in the third trimester (figure 2).

This study is important in illustrating how we can combine data from multiple studies to improve our knowledge base. As Leeson comments in the accompanying editorial (see page 490):" "The authors should be commended for addressing this relevant subject and their use of a meta-analysis to generate findings from a larger scale dataset. Longitudinal studies in pregnant populations are challenging to undertake. Harmonisation of results from available research cohorts to consolidate our understanding of this time of unique physiological challenge is potentially a powerful way to accelerate research knowledge and gain insight into cardiovascular reserve during pregnancy".

The risk of hypertension is higher in people with greater blood pressure reactivity to psychological stress. Crump and colleagues (see page 541) hypothesized that this effect is important over the lifetime of the patient such that low stress resilience early in life will be associated with hypertension later in life. In about 1.5 million military conscripts in Sweden, stress resilience measured at age 18 years was compared to the risk of hypertension at a median follow-up of 25.7 years. Stress resilience was measured on a 1 (low) to 9 (high) scale based on a 30-minute structured interview administered by trained psychologists. This data showed that low stress resilience at age 18 was associated with increased risk of later hypertension (lowest vs highest quintile: HR $1.43 ; 95 \%$ CI 1.40 to 1.46 ; $\mathrm{p}<0.001$ ), even after adjustment for body

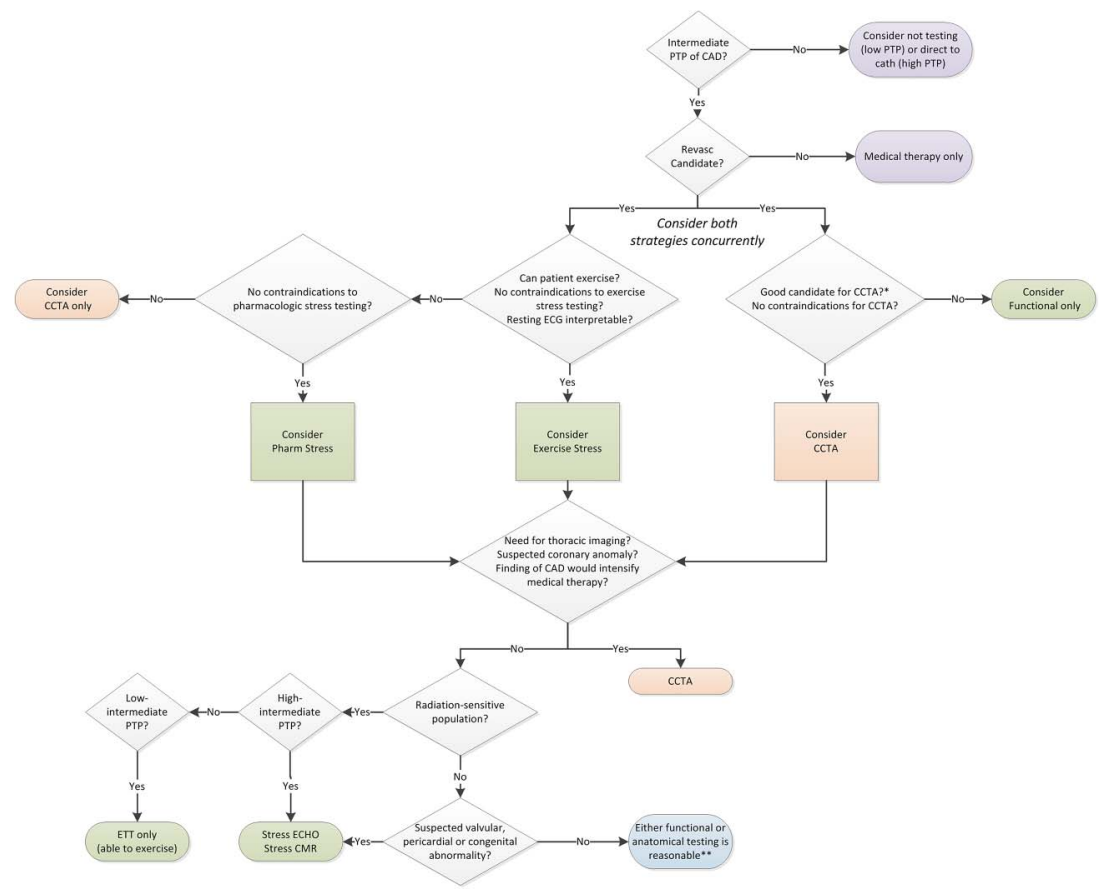

Figure 4 Proposed integrated approach to initial non-invasive test selection using both functional and anatomical approaches for the diagnosis of IHD in stable chest pain patients. ${ }^{*}$ See text; ${ }^{* *}$ Consider exercise treadmill test (ETT) or CCTA for low-intermediate pretest probability (PTP); consider stress echo, myocardial perfusion imaging (MPI) or cardiovascular MR (CMR) for high-intermediate PTP. CAD, coronary artery disease. mass index (BMI), family history and socioeconomic factors. These risk ratios correspond to incidence rates ranging from 278.7 to 180.0 per 100000 personyears (figure 3). An increased BMI further increased risk, with a more than 3-times higher risk of hypertension for the combination of low stress resilience and high BMI.

The mechanisms of the association between stress resilience in young adulthood and hypertension in later life are complex, reflecting both pathophysiological changes and associated lifestyle behaviors. Reduced stress resilience is associated with increased blood pressure reactivity which may affect arterial structure and function in the long term. In addition, people with low stress resilience are more likely to engage in unhealthy behaviors such as physical inactivity and smoking, as well being prone to anxiety and depression. The authors suggest that we focus on prevention, not just treatment, of hypertension including efforts to improve stress resilience in young men, particularly those with a high BMI who are at greatest risk. Additional studies in young women would be of interest to see if the effect of stress resilience is similar to that seen in men.

The Education in Heart article in this issue of Heart (see page 555) discusses selection of the best non-invasive imaging test for diagnosis of ischemic heart disease. A practical algorithm is provided along with a comparison of recommendations from the American College of Cardiology/American Heart Association, the European Society of Cardiology and the UK National Institute for Health and Care Excellence.

Try the Image Challenge (see page 540) to diagnose the cause of chest pain in a 39 year old woman based on the chest radiograph and CT scan. If you disagree or have a comment, click the "submit a response" link on the online menu for the full text version of the article on the Heart website (http://heart.bmj.com/).

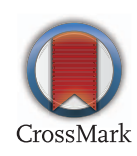

To cite Otto CM. Heart 2016;102:485-486.

Heart 2016;102:485-486.

doi:10.1136/heartjnl-2016-309537 